\title{
FIRST-PRINCIPLES STUDY OF InAs/GaAs(001) HETEROEPITAXY
}

\author{
Evgeni Penev and Peter Kratzer \\ Fritz-Haber-Institut der Max-Planck-Gesellschaft \\ Faradayweg 4-6, D-14195 Berlin-Dahlem, Germany
}

\begin{abstract}
Density-functional theory calculations are employed to obtain important information about the morphology of III-V semiconductor surfaces and kinetics of epitaxial growth. In this way, insight into the microscopic processes governing quantum dot formation in $\operatorname{InAs} / \mathrm{GaAs}(001)$ heteroepitaxy is gained. First, we investigate theoretically the atomic structure and thermodynamics of the wetting layer formed by InAs deposition on $\operatorname{GaAs}(001)$, including the effect of strain in our discussion. Secondly, we present results about In adatom diffusion both on the wetting layer and on the $\mathrm{c}(4 \times 4)$-reconstructed $\mathrm{GaAs}(001)$ surface. In the latter case, we demonstrate the importance of mechanical stress for the height of surface diffusion barriers. Implications for the growth of InAs quantum dots on $\operatorname{GaAs}(001)$ are discussed.
\end{abstract}

\section{Introduction}

Self-organized quantum-dot heterostructures $[1,2]$, or simply quantum dots (QDs), will soon leave their "teens" behind. The high technological promises they have brought to optoelectronics have triggered an enormous scientific activity. As a representative example one can take the work on the InAs/GaAs lattice-mismatched heteroepitaxial system, Fig. 1. In terms of the observed resultant morphology, the epitaxy of QDs (e.g. molecular beam epitaxy (MBE), or metal-organic chemical vapor deposition (MOCVD)) follows the Stranski-Krastanov growth mode [3]. Though our knowledge on the nature of the StranskiKrastanov regime has considerably increased, both experimentalists and theorists are still on the way to complete understanding the intricacies of QD growth kinetics. System-specific, microscopic information in this respect is a must. Important information comes from direct experimental probes, like in situ scanning tunnelling microscopy (STM), reflection high-energy electron diffraction (RHEED), or reflectance-difference spec- 


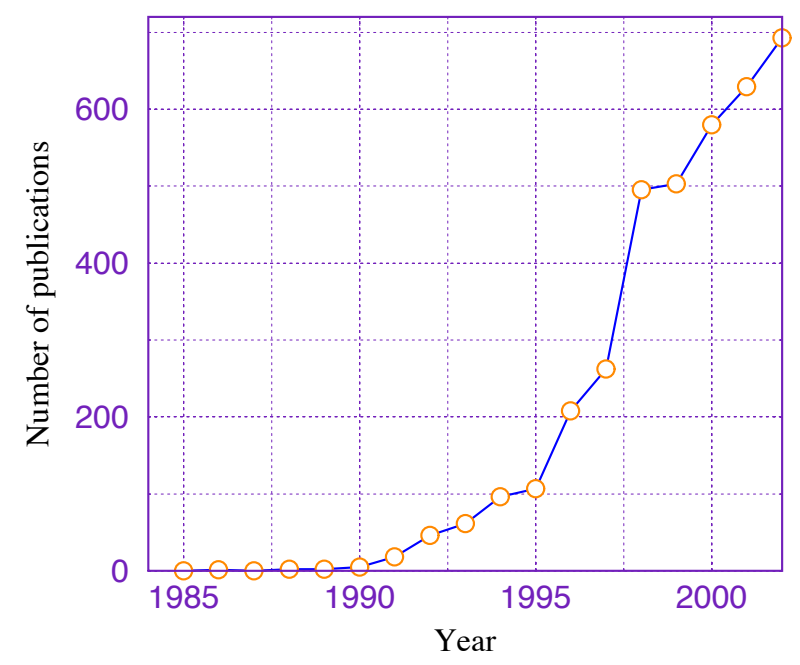

Figure 1. Number of articles published in the period 1985-2002, containing the terms 'quantum $\operatorname{dot}(\mathrm{s})$ ', and 'InAs' or 'GaAs' in any of the title, abstract or the list of keywords according to Science Citation Index Expanded (isiknowledge.com).

troscopy (RDS). However, if one is aiming at a complete understanding of $\mathrm{QD}$ growth, the information accessible from experiments may not be sufficient. This is particularly true if one focuses on the characteristics of species dynamics at the surface, details of the surface atomic structure, thermodynamic quantities, etc.

This contribution addresses InAs heteroepitaxy on GaAs(001) - the III-V semiconductor system of ultimate importance for QD "self-fabrication" [4]. An extensive track record of experimental studies has established a number of anomalies in the QD self-assembly for this system [5]. It was also brought out clearly that any theory has to capture the subtleties of strain-dependent QD growth kinetics [6]. Thus the first fundamental questions to be answered are how strain affects surface morphology, and its impact on surface adatom mobility. The characteristic length and time scales for the latter lie in the microscopic range, 0.1$10 \AA$ and $10^{-15}-10^{-13} \mathrm{~s}$, where the density-functional theory (DFT) is one of the most commonly used theoretical tools $[7,8]$. By considering a few case studies we shall attempt to give an overview of an appropriate theoretical framework, building on DFT, to tackle the above mentioned problems in InAs/GaAs(001) heteroepitaxy. We feel it however compelling to note that its applicability is far more broader and has now covered problems in catalysis, biochemistry, etc. [9]. 
We start with a brief description of the methodology used to calculate the quantities of interest, e.g. surface energies and adatom diffusion barriers. Then the effect of strain on the equilibrium surface reconstructions of $\operatorname{GaAs}(001)$ and $\operatorname{InAs}(001)$ is discussed. Analysis of the strain dependence of indium diffusivity is considered as well. Finally, a brief outlook is provided.

\section{Theoretical Framework}

\subsection{First-Principles Surface Thermodynamics}

Understanding the properties of the InAs/GaAs(001) heteroepitaxial system naturally implies detailed knowledge of the surface properties of both subsystems. Thus, as a first step, let us consider the equilibrium structures of $\operatorname{GaAs}(001)$ and $\operatorname{InAs}(001)$. The key quantity determining the most stable surface reconstruction under given growth conditionstemperature $T$ and pressure $p$-is the surface free energy $\gamma$, defined as

$$
\gamma=\frac{1}{A}\left[G\left(p, T,\left\{N_{i}\right\}\right)-\sum_{i} \mu_{i} N_{i}\right] .
$$

Here, $A$ is the surface area, $\mu_{i}$ is the chemical potential of the $i$ th component, $N_{i}$ the corresponding number of particles, and $G\left(p, T,\left\{N_{i}\right\}\right)$ is the Gibbs free enthalpy. For a GaAs surface Eq. (1) translates into

$$
\gamma A=G\left(p, T, N_{\mathrm{Ga}}, N_{\mathrm{As}}\right)-\mu_{\mathrm{Ga}} N_{\mathrm{Ga}}-\mu_{\mathrm{As}} N_{\mathrm{As}} .
$$

Exploiting the fact that in thermodynamic equilibrium

$$
\mu_{\mathrm{Ga}}+\mu_{\mathrm{As}}=\mu_{\mathrm{GaAs}}(p, T),
$$

and that in MBE As-rich growth conditions are typical for the case at hand, one can eliminate $\mu_{\mathrm{Ga}}$, which leads to

$$
\gamma A=G\left(p, T, N_{\mathrm{Ga}}, N_{\mathrm{As}}\right)-\mu_{\mathrm{GaAs}}(p, T) N_{\mathrm{Ga}}-\mu_{\mathrm{As}} \Delta N,
$$

where $\Delta N=N_{\mathrm{As}}-N_{\mathrm{Ga}}$ is the surface stoichiometry. The chemical potential $\mu_{\mathrm{As}}$ can take on values in the range

$$
\mu_{\mathrm{As}(\mathrm{bulk})}(p, T)-\Delta H_{\mathrm{GaAs}}(p, T) \leq \mu_{\mathrm{As}} \leq \mu_{\mathrm{As}(\text { bulk })}(p, T),
$$

$\Delta H_{\mathrm{GaAs}}$ being the heat of formation of GaAs. It should be noted, however, that one can work out all terms in Eqs. (4) and (5) from a groundstate formalism only for $(p, T)=0$. Nonetheless, while thermodynamic stability in general requires a discussion of the free energy, it has been 
established in the literature $[10,11,12,13,14,15]$ that the stability of surface reconstructions is dominated by the (static) energy differences of relaxed structures. For a discussion of surface energies, it is in general adequate to neglect contributions to the free energy coming from the kinetic energy of the atoms, as well as from entropy terms. This is due to the fact that the surface free energy is the difference between two free energies, of the semi-infinite solid plus its surface on the one hand side, and of the reservoirs for the constituent atomic species (gas phase, elemental bulk phases) on the other. By taking the difference between these free energies, the kinetic and entropic terms largely cancel.

With this remark, Eqs. (4) and (5) can be transcribed in a form which provides a direct link to ab initio calculations:

$$
\begin{gathered}
\gamma A=E^{\mathrm{tot}}\left(N_{\mathrm{Ga}}, N_{\mathrm{As}}\right)-N_{\mathrm{Ga}} E_{\mathrm{GaAs}}^{\mathrm{tot}}-\Delta N\left[\mu_{\mathrm{As}}-\mu_{\mathrm{As}(\mathrm{bulk})}(0,0)\right], \\
-\Delta H_{\mathrm{GaAs}}(0,0) \leq \mu_{\mathrm{As}}-\mu_{\mathrm{As}(\text { bulk })}(0,0) \leq 0 .
\end{gathered}
$$

In these expressions, $E^{\text {tot }}\left(N_{\mathrm{Ga}}, N_{\mathrm{As}}\right)$ is the total energy of the system representing the GaAs surface, $E_{\mathrm{GaAs}}^{\mathrm{tot}}$ the total energy per Ga-As pair in bulk GaAs, $\mu_{\mathrm{As}(\mathrm{bulk})}(0,0)=E_{\mathrm{As}}^{\text {tot }}$ the total energy per atom in bulk As metal, and

$$
\Delta H_{\mathrm{GaAs}}(0,0)=E_{\mathrm{GaAs}}^{\mathrm{tot}}-E_{\mathrm{Ga}}^{\mathrm{tot}}-E_{\mathrm{As}}^{\mathrm{tot}} .
$$

All $E^{\text {tot }}$ contributions can now be obtained from DFT calculations. Our setup [17] employs norm-conserving pseudopotentials in conjunction with a plane-wave basis set (with a cutoff energy of $10 \mathrm{Ry}$ ) and the local-density approximation to the electronic exchange and correlation energy. Surfaces are represented, within the supercell approximation, by slabs of eight (or seven) atomic layers whose bottom (cationterminated) surface is passivated [18] by pseudo-hydrogen $\left(\mathrm{H}^{*}\right)$ atoms $(Z=1.25)$. Brillouin zone (BZ) integration is carried out using a set of special k-points equivalent to at least 64 points in the $1 \times 1$ surface BZ. The presence of $\mathrm{H}^{*}$ in the actual calculations entails a slight modification of Eq. (6). In order to subtract the contribution of the $\mathrm{H}^{*}$ passivated side of the slab, a similar calculation is performed for a slab with two $\mathrm{H}^{*}$-passivated surfaces, whereby we replace $E^{\text {tot }}\left(N_{\mathrm{Ga}}, N_{\mathrm{As}}\right) \rightarrow$ $E_{\text {slab }}^{\text {tot }}\left(N_{\mathrm{Ga}}, N_{\mathrm{As}}, N_{\mathrm{H}^{*}}\right)-\frac{1}{2} E_{\mathrm{H}^{*}-\text { slab }}^{\text {tot }}\left(N_{\mathrm{Ga}}^{\prime}, N_{\mathrm{As}}^{\prime}, N_{\mathrm{H}^{*}}\right)$. The complete energy "accounting" is exemplified for the $\beta 2(2 \times 4)$-reconstructed $\mathrm{GaAs}(001)$ surface [19] in Fig. 2. Further technical provisions can be found, for examples, in Refs. $[10,20]$. This scheme can be readily generalized to the case where surfaces are under strain as well as to account for a possible formation of a ternary surface alloy during the initial stages of InAs deposition on $\operatorname{GaAs}(001)$. 


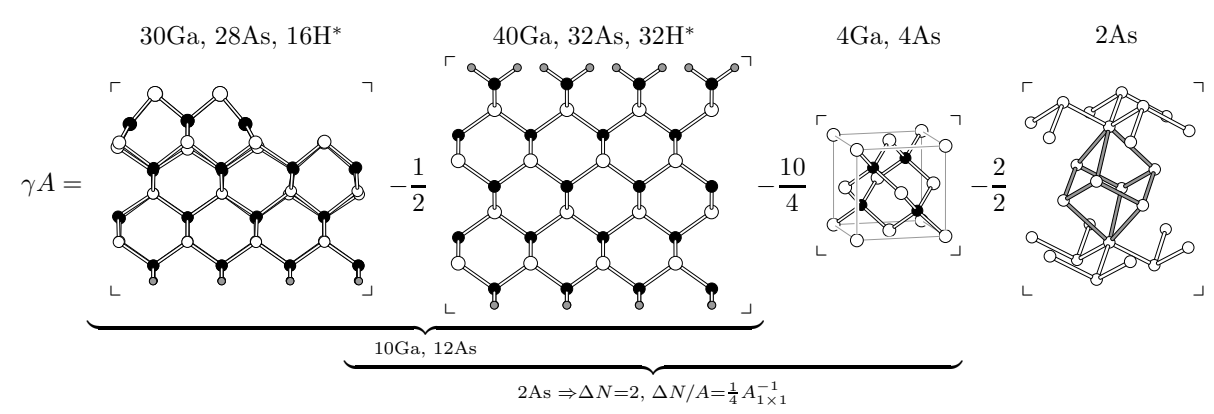

Figure 2. Pictorial representation of the procedure for calculating the surface energy of $\operatorname{GaAs}(001)-\beta 2(2 \times 4)$. Number of atoms per species in the supercell used in the actual calculation is given above each system. Total energies of the corresponding systems are symbolically given by small corners. The As reservoir is taken to be rhombohedral bulk As metal - the so-called A7 structure [16].

\subsection{Surface Diffusion}

Although an equilibrium description may be applicable in some cases of III-V epitaxy [21, 22], often thermodynamic equilibrium is not established during growth. Knowledge of kinetic parameters is then crucial in finding a rationale for the observed growth morphology.

In the MBE growth of arsenide compound semiconductors it is the surface diffusion that mainly governs the incorporation of the cation species, e.g. Ga, In, Al, whereas the kinetics of arsenic incorporation is dominated by adsorption/desorption of $\mathrm{As}_{2}$ or $\mathrm{As}_{4}$ molecules at surface sites with enhanced local population of cations [8] - an observation dating back to the dawn of MBE experiments [23].

Typically, surface adatom mobility can be understood on the basis of the adatom's binding specifics at the surface accessible from the relevant potential-energy surface(s) (PES). This energy "map" is defined as the minimized difference

$$
E_{b}(X, Y)=\min _{\mathbf{R}^{\prime} \subseteq \mathbf{R}} \min _{Z} E^{\mathrm{tot}}\left(\mathbf{R}, \mathbf{R}^{\mathrm{ad}}\right)-\mathbf{E}_{\mathrm{slab}}^{\mathrm{tot}}-\mathbf{E}_{\text {atom }} .
$$

In this expression $E^{\text {tot }}\left(\mathbf{R}, \mathbf{R}^{\text {ad }}\right)$ stands for the total energy of the adatom/ surface system; $E_{\text {slab }}^{\text {tot }}$ and $E_{\text {atom }}$ are those of the bare surface (modeled by a slab) and the isolated atom, respectively. Minimization is carried out with respect to the height of the adatom $Z$, and a given subset $\mathbf{R}^{\prime}$ or eventually all coordinates $\mathbf{R}$ of the substrate atoms, $\mathbf{R}^{\text {ad }}=(X, Y, Z)$ being the adatom coordinates. Further details can be found, e.g. in Refs. [10, 20, 24]. 

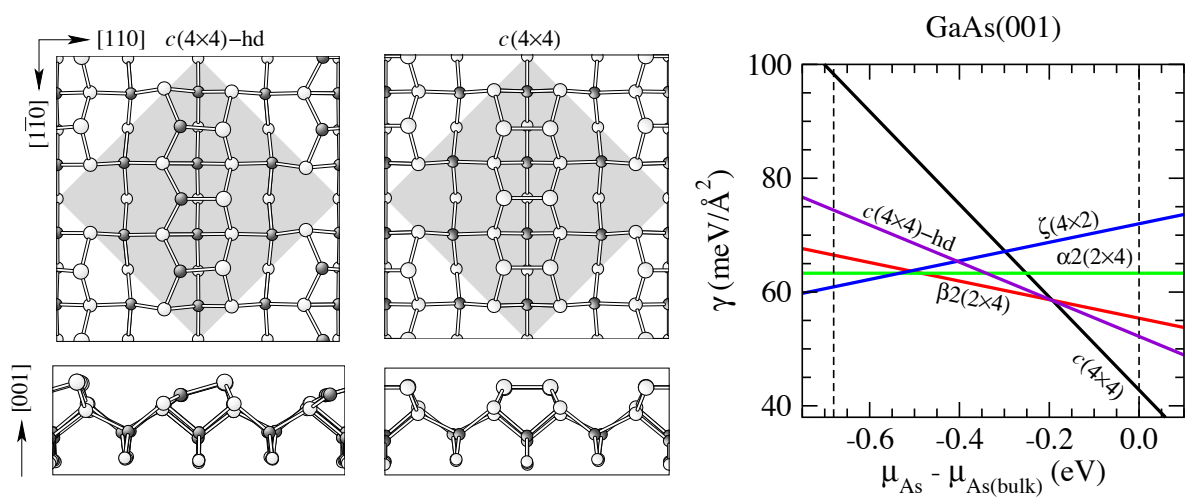

Figure 3. Equilibrium phase diagram of the GaAs(001) surface (right panel) calculated within the local-density approximation. Dashed lines mark the physically allowed range of variation of $\mu_{\mathrm{As}}$ as determined from Eqs. (7) and (8). Structural models (topmost 4 atomic layers) of the conventional $\mathrm{c}(4 \times 4)$ reconstruction is shown on the middle, and the $\mathrm{c}(4 \times 4)$-hd heterodimer model is shown on the left (Ga: shaded atoms; As: bright atoms). Shaded squares represent the surface unit cell.

\section{Surface Phases under Strain}

\subsection{Clean $\operatorname{GaAs}(001)$ and $\operatorname{InAs}(001)$ Surfaces}

Recent theoretical work $[20,25,26,27,28,29]$ has established that for growth conditions ranging from very As-rich to very Ga-rich the stable reconstructions of the $\operatorname{GaAs}(001)$ and $\operatorname{InAs}(001)$ surfaces follow the sequence $\mathrm{c}(4 \times 4), \beta 2(2 \times 4), \alpha 2(2 \times 4)$, and $\zeta(4 \times 2)$. The calculated surface phase diagram of $\operatorname{GaAs}(001)$, for example, is shown in Fig. 3. However, so far only partial information is available about the influence of strain on the stability of these reconstructions (for $\operatorname{InAs}(001)$ see Ref. [30]). Furthermore, a very recent experiment [31] has also uncovered a new structural model for the $\mathrm{c}(4 \times 4)$ reconstruction in the case of GaAs(001), see Fig. 3. To clarify this issue, we have performed calculations of the surface free energy for all four reconstructions for isotropically strained (001) substrates [29]. For small strain values $\epsilon$ one can write $\gamma$ in the form

$$
\gamma(\epsilon)=\gamma(0)+\operatorname{Tr}(\boldsymbol{\tau}) \epsilon+\mathrm{O}\left(\epsilon^{2}\right)
$$

where $\boldsymbol{\tau}$ is the intrinsic surface stress tensor [32]. From the slope of $\gamma(\epsilon)$ at $\epsilon=0$, we conclude that $\operatorname{Tr}(\boldsymbol{\tau})$ is positive for all reconstructions of both $\operatorname{GaAs}(001)$ and $\operatorname{InAs}(001)$. In Fig. 4, we show the regions of stability of the reconstructions in the $\left(\mu_{\mathrm{As}}, \epsilon\right)$ plane. For GaAs(001), at positive $\epsilon$ 

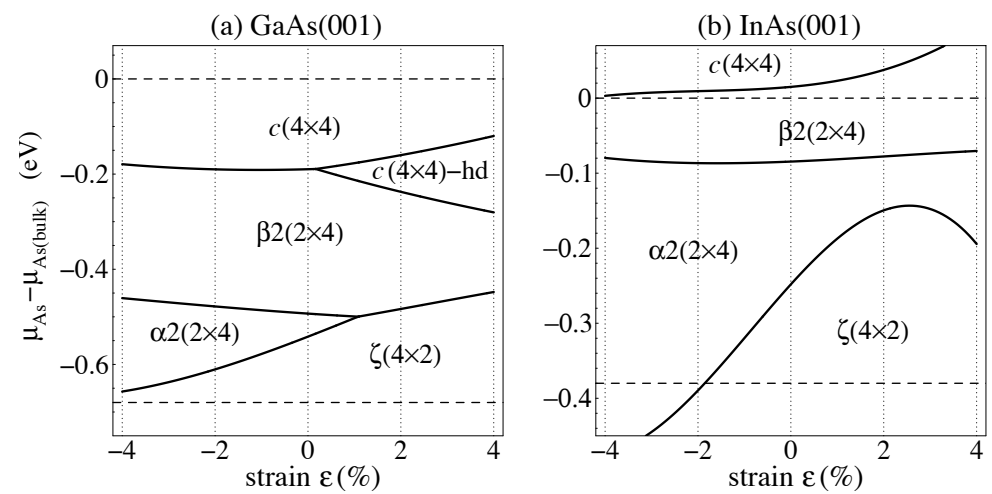

Figure 4. Diagrams of surface phases of (a) GaAs(001), and (b) InAs(001) surfaces as a function of $\mu_{\mathrm{As}}$ and isotropic strain $\epsilon$. Dashed lines mark the physically allowed range of variation of $\mu_{\mathrm{As}}$.

the "heterodimer" $\mathrm{c}(4 \times 4)$ model, hereafter referred to as $\mathrm{c}(4 \times 4)-\mathrm{hd}$, appears as a stable reconstruction between the conventional $\mathrm{c}(4 \times 4)$ and $\beta 2(2 \times 4)$ reconstructions. In MBE of InAs on GaAs, typically performed under As-rich conditions, the $\operatorname{GaAs}(001)$ surface displays the $\mathrm{c}(4 \times 4)$ reconstruction, Fig. 3 , which is characterized by blocks of three As dimers, or three Ga-As heterodimers, sitting on top of a complete As layer.

It is also remarkable that the stability range of $\zeta(4 \times 2)$ increases noticeably for $\epsilon>0$, especially for $\operatorname{InAs}(001)$, while the $\alpha 2(2 \times 4)$ range increases for large negative strain. The latter trend is in agreement with the results of Ref. [30], although quantitative differences can be noticed. Thus, one could expect the $\operatorname{InAs}(001)$ surface at the heteroepitaxial strain of $\simeq-7 \%$ to expose the $\alpha 2(2 \times 4)$ reconstruction over nearly the whole range of $\mu_{\mathrm{As}}$.

\subsection{Wetting Layer}

The initial delivery of InAs to the GaAs(001) substrate leads to formation of a microscopic pseudomorphic film, the so-called wetting layer (WL). Experimentally it is found that a complete layer structurally different from the substrate appears already after deposition of $1 / 3-2 / 3 \mathrm{ML}$ of InAs, depending on $T$. The appearance of this new structure is accompanied by a change in the RHEED pattern which gives evidence for a period of three lattice constants in the [110] direction. Other probes, such as RDS [33, 34], STM and X-ray diffraction, confirm this structural transition $[35,36]$. However, there is also evidence for some asymme- 

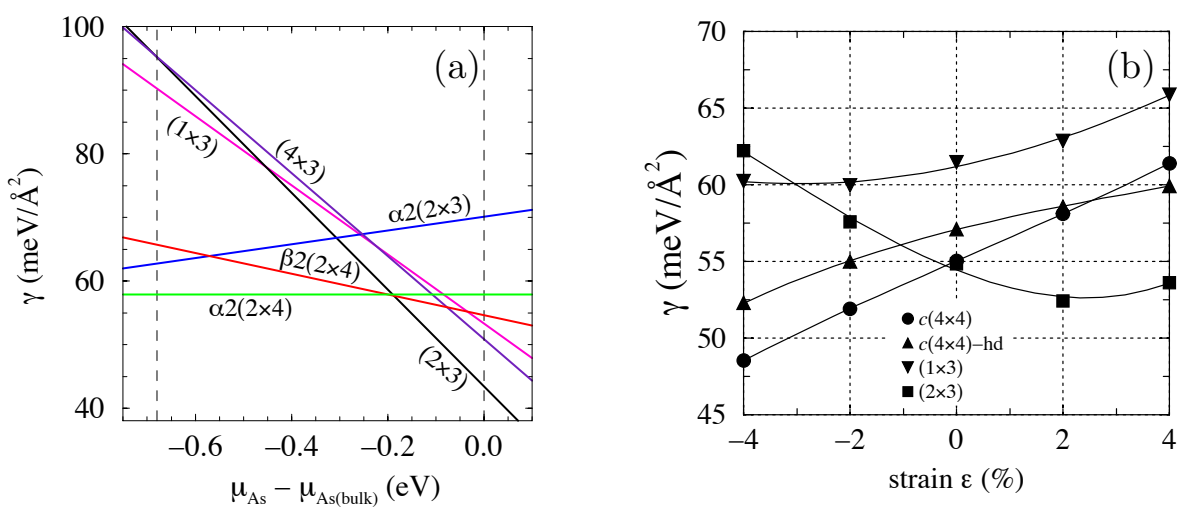

Figure 5. (a) Formation energy of $\mathrm{In}_{2 / 3} \mathrm{Ga}_{1 / 3} \mathrm{As}(001)$ film with different reconstructions as a function of $\mu_{\mathrm{As}}$, cf. Ref. [15]. (b) Surface energy of GaAs(001)-c(4×4) and energy of formation of $(1 \times 3)$ - and $(2 \times 3)$-reconstructed $\operatorname{In}_{2 / 3} \mathrm{Ga}_{1 / 3} \mathrm{As}(001)$ film as functions of isotropic strain $\epsilon$ for $\mu_{\mathrm{As}}-\mu_{\mathrm{As}(\mathrm{bulk})}=-0.15 \mathrm{eV}$. The lines are polynomial fits to the calculated points [29].

try/disorder in the diffraction patterns. A rationale for the asymmetry and the triple periodicity has been proposed a long time ago [37], based on domain formation.

Here we explore the appearance of the $\times 3$ periodicity as directly related to formation of a ternary InGaAs surface alloy with this specific surface unit cell. For InAs deposition of $2 / 3 \mathrm{ML}$, a structural model with a $(2 \times 3)$ unit cell (see Fig. $6(\mathrm{~b})$ below) has been suggested on the basis of X-ray diffraction data [36, 38]. It consists of (continuous) rows of As dimers running in the [110] direction, similar to the $\mathrm{c}(4 \times 4)$ reconstruction of pure $\operatorname{GaAs}(001)$. The indium atoms are located solely on lattice sites in the "trenches" between the chains of As dimers, avoiding the lattice sites directly below the As dimers. Thus one could speak of this film as a single ML of $\operatorname{In}_{2 / 3} \mathrm{Ga}_{1 / 3}$ As alloy. Alternatively, a $(1 \times 3)$ model has been also proposed [34].

The calculated formation energy, Fig. 5 (a), for the $(2 \times 3)$ structural model is found to be very close to the surface energy of the $\mathrm{c}(4 \times 4)$ substrate, over a wide range of $\mu_{\mathrm{As}}$. For $-0.2 \mathrm{eV}<\mu_{\mathrm{As}}-\mu_{\mathrm{As} \text { (bulk) }}<$ $-0.1 \mathrm{eV}$, the $(2 \times 3)$ structure is slightly more stable. We thus confirm the latter as the basic subunit of the WL. The $(1 \times 3)$ and other variants with different periodicity in the [110] direction, see Fig. 5 (a), turn out to be higher in energy and can be discarded [15].

Another important feature of the pseudomorphic $\mathrm{In}_{2 / 3} \mathrm{Ga}_{1 / 3} \mathrm{As}(001)$ film shows up upon considering $\gamma$ as a function of the applied isotropic strain $\epsilon$. From the slope of $\gamma$ at $\epsilon=0$ we infer that the dominant $\tau_{\alpha \beta}$ com- 
ponent is compressive, $\operatorname{Tr}(\boldsymbol{\tau})<0$, in contrast to those of pure $\mathrm{GaAs}(001)$ and $\operatorname{InAs}(001)$, as well as $\operatorname{InGaAs}(001)-(1 \times 3)$. In Fig. 5 (b), we compare the formation energy of the WL with the surface energy of the bare $\operatorname{GaAs}(001)-c(4 \times 4)$ substrate. The different surface stress of these surfaces renders the bare substrate more stable at compressive strain, while the $(2 \times 3)$ film is preferred for $\epsilon>0$. We do not find conditions which render the $(1 \times 3)$ or the $\mathrm{c}(4 \times 4)$-hd reconstructions the most stable. Consequently, thermodynamics combined with our first-principles results imply suppression of wetting for $2 / 3 \mathrm{ML}$ of InAs on the GaAs if the substrate is (locally) under mechanical compression, while the same amount of deposited InAs is sufficient to form a homogeneous WL on an unstrained substrate.

\section{Surface Diffusion of Indium}

Consider now the problem of indium migration in the process of InAs deposition on $\operatorname{GaAs}(001)$. In Ref. [42] we have already studied in great detail In diffusion on the $\operatorname{GaAs}(001)-\mathrm{c}(4 \times 4)$ surface. It is governed by the PES shown in Fig. 6 (a), from which one finds that the strongest binding site $\left(\mathbf{A}_{\mathbf{1}}\right)$ is located at the missing dimer position in the $\mathrm{c}(4 \times$ 4) structure. In a first approximation, the adatom migration can be described as jumps between the $\mathbf{A}_{\mathbf{1}}$ sites crossing the saddle points $\mathbf{T}_{\mathbf{1}}$ leading to an isotropic diffusion characterized with energy barrier of $\Delta E=0.65 \mathrm{eV}$. At higher temperatures, a slight anisotropy may result upon inclusion of longer jumps across the blocks of 3 As dimers, passing the $\mathbf{T}_{\mathbf{2}}-\mathbf{A}_{\mathbf{2}}-\mathbf{T}_{\mathbf{2}}$ sites, thus increasing the indium diffusion coefficient along the [110] direction. Let us note that the $\mathrm{In} / \mathrm{GaAs}(001)-\mathrm{c}(4 \times 4)$ system is suitable for modeling the arrival of the first In atom to the GaAs surface in the initial stages of InAs deposition (or eventually to the GaAs capping layer in growth of multilayer QD structures), but the results cannot be taken over directly to the later stages of island growth.

As a next step we focus on In migration under those conditions where alloying in the wetting layer leads to a surface atomic arrangement with threefold periodicity along the [110] surface direction. More precisely, we have studied surface diffusion on the $\operatorname{InGaAs}(001)-(2 \times 3)$ pseudomorphic film. While the latter may not exactly represent the typical wetting layer encountered in experiments [39, 40], we can learn from this system about the effect of alloying on the diffusivity by comparing to the case of In diffusion on $\mathrm{GaAs}(001)-\mathrm{c}(4 \times 4)$. It can be viewed as a particular case of surface diffusion where the adatom migrates on a homogeneously strained submonolayer film. 

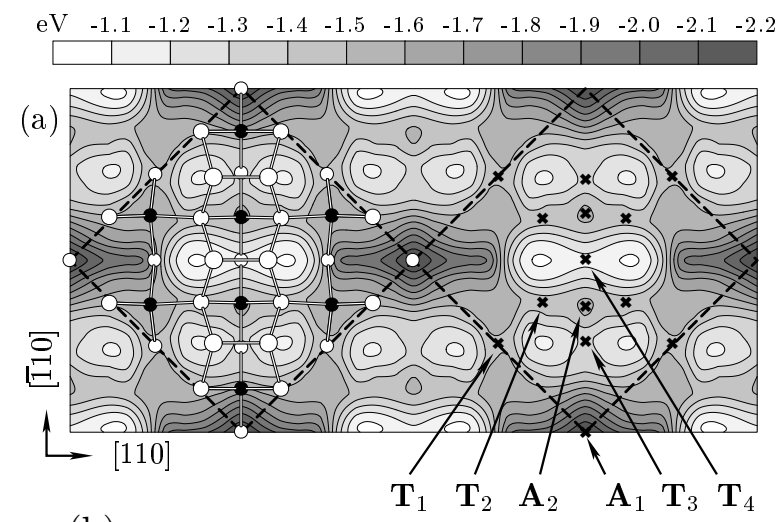

(b)

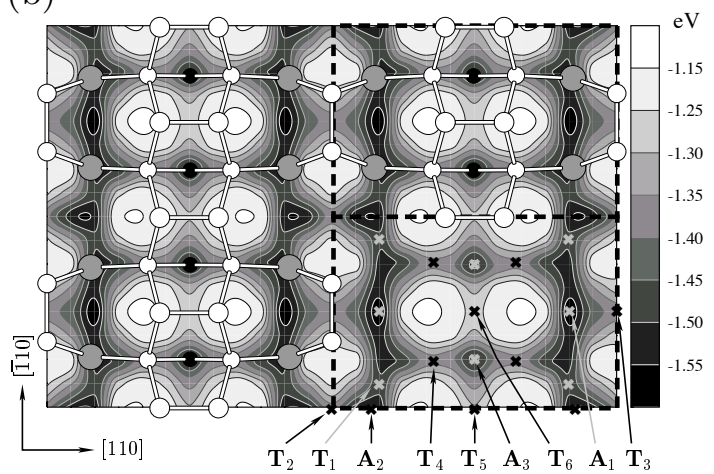

Figure 6. (a) PES for an In adatom on the GaAs(001)-c(4×4) surface, cf. Ref. [42]. Atomic positions in the clean surface unit cell are indicated for atoms in the upper four layers (As: empty circles; Ga: filled circles). (b) PES for an In adatom on the $\mathrm{In}_{2 / 3} \mathrm{Ga}_{1 / 3} \mathrm{As}(001)-(2 \times 3)$ surface. Two unit cells are indicated by dashed boxes. Overlaid on the PES plot are the topmost 3 atomic layers (In: shaded circles).

The PES for an indium adatom on the $\operatorname{In}_{2 / 3} \mathrm{Ga}_{1 / 3} \mathrm{As}(001)-(2 \times 3)$ surface is shown in Fig. 6 (b). The corrugation of this PES is remarkably small: The maximum variation of the adiabatic potential in the (001) plane is $\simeq 0.5 \mathrm{eV}$. We find 3 symmetry-inequivalent potential minima and 6 saddle points: The energy barriers are all smaller than $0.3 \mathrm{eV}$. The adsorption site providing strongest binding, $\mathbf{A}_{\mathbf{1}}$, is located between a top-layer As dimer and the As dimer bound to the third-layer In atoms. Another adsorption site, $\mathbf{A}_{\mathbf{2}}$, appears next to a top-layer As dimer, but located in the gap between two As dimers bound to the third-layer In atoms. The troughs in the continuous As dimer row in [110] direction give rise to a shallower site, $\mathbf{A}_{\mathbf{3}}$. Detailed analysis of In diffusivity will be given elsewhere. 

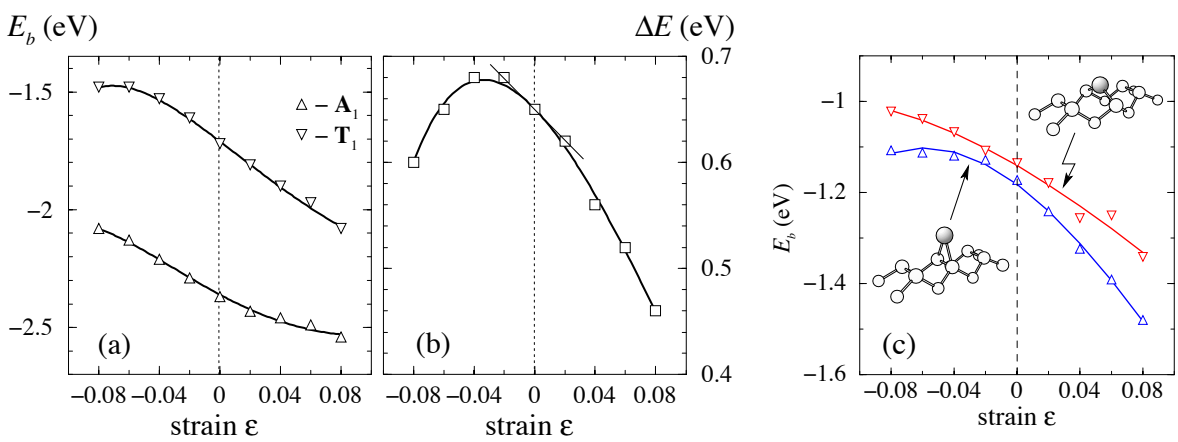

Figure 7. (a) Binding energy $E_{b}$ as a function of isotropic strain $\epsilon$ for an indium adatom at the $\mathbf{A}_{1}$ and $\mathbf{T}_{1}$ sites, cf. Fig. 6 (a). (b) diffusion barrier $\Delta E \equiv E_{b}\left(\mathbf{T}_{1}\right)-E_{b}\left(\mathbf{A}_{1}\right)$ as a function of $\epsilon$. Full curves on both panels represent leastsquares polynomial fits to the calculated points [42]. (c) Binding energy of an indium adatom for the depicted bonding configurations.

Thus the typical energy scales for the potential-energy surfaces on the bare substrate and the pseudomorphic film turn out clearly different. If we define an onset temperature for diffusion by demanding that a single jump should occur at least once per second, the onset of In diffusion on the $\mathrm{In}_{2 / 3} \mathrm{Ga}_{1 / 3} \mathrm{As}(001)$ film in the [110] direction occurs at a temperature about $130 \mathrm{~K}$ lower than on the $\mathrm{GaAs}(001)$-c $(4 \times 4)$ surface. For diffusion in the [110] direction, the onset temperature is even lower by $190 \mathrm{~K}$, compared to the $\operatorname{GaAs}(001)-\mathrm{c}(4 \times 4)$ surface. As we have not found evidence for the so-called compensation effect [41] on the attempt frequencies, one can predict a considerably higher In mobility on the $\mathrm{In}_{2 / 3} \mathrm{Ga}_{1 / 3} \mathrm{As}(001)$ film as compared to the $\operatorname{GaAs}(001)-\mathrm{c}(4 \times 4)$ substrate.

Additionally, we have discovered [42] that In diffusivity on the $\mathrm{c}(4 \times 4)$ reconstructed GaAs substrate is considerably affected by the presence of mechanical strain. This situation is pertinent, e.g. to indium diffusion on a GaAs capping layer with buried QDs, acting as stressors. The strain dependence of the adatom binding at the adsorption sites $\mathbf{A}_{\mathbf{1}}$ and the lowest saddle point connecting them, $\mathbf{T}_{\mathbf{1}}$, is shown in Fig. 7 (a). While for $\epsilon<0 E_{b}$ at the adsorption site $\mathbf{A}_{1}$ follows approximately a linear law with a slope of $-3.8 \mathrm{eV}$, the binding energy at $\mathbf{T}_{1}$ contains small non-linear terms in strain which do not cancel in the evaluation of the diffusion barrier $\Delta E=E_{b}\left(\mathbf{T}_{1}\right)-E_{b}\left(\mathbf{A}_{1}\right)$. Thus, $\Delta E$ for $\epsilon<0$ is accurately described by

$$
\Delta E(\epsilon)=0.65 \mathrm{eV}+0.03 \mathrm{eV} \times\left[1-\left(\frac{\epsilon}{0.03}+1\right)^{2}\right], \quad \epsilon<0 .
$$




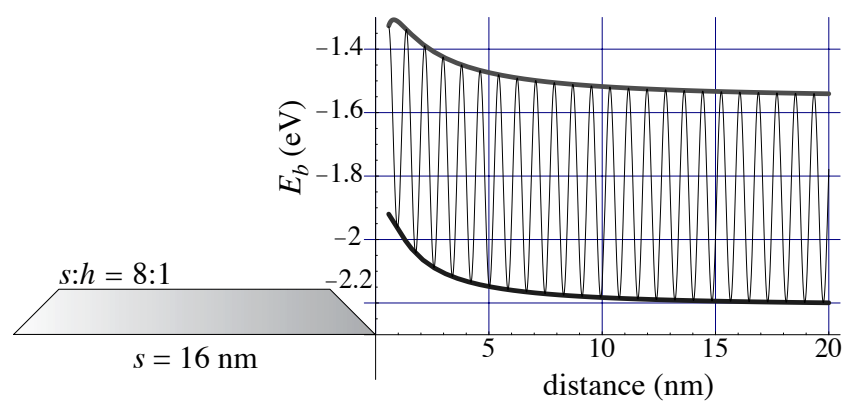

Figure 8. Migration potential (oscillating curve) for an In adatom approaching perpendicular to a very long, coherently strained InAs island (of width $s$ and height $h)$ on the $\mathrm{c}(4 \times 4)$-reconstructed $\operatorname{GaAs}(001)$ surface. In addition to the diffusion potential due to the atomic structure of the surface, the strain field in the substrate induced by the island gives rise to a repulsive potential that lifts both the binding energies (thick lower line) and transition state energies (thick upper line) close to the island, cf. Ref. [46]

It should be noted, however, that for small values of the strain, the diffusion barrier is to a very good accuracy linear in the strain [43, 44], $\Delta E \propto \epsilon$, as can be seen from $\Delta E(\epsilon)$ in Fig. 7 (b) in the range $\pm 2 \%$. For an inhomogeneously strained sample, the pronounced strain dependence of $E_{b}$ for both the adsorption site and the saddle point will introduce a position dependence of $\Delta E$.

In order to illustrate this effect we have considered, within the flatisland approximation [45], a simple model $[20,46]$ for an InAs island on a $\operatorname{GaAs}(001)-c(4 \times 4)$ substrate, Fig. 8 . As can be seen from the figure, the effect of strain leads to a repulsive potential with a strength of up to $0.2 \mathrm{eV}$, that affects both the binding energy and, to a slightly smaller extent, the diffusion barriers for an In adatom that attempts to approach this island. This repulsive interaction can significantly slow down the speed of growth of strained islands. For a simple example, where two very elongated islands compete for the flux of In atoms deposited between them, we have found that strain-controlled diffusion indeed tends to equalize the size of the two islands while they are growing [42]. This effect has been proposed as one of the factors responsible for the narrowing of the island size distribution that is desirable from the point of view of the applications $[47,48]$.

\section{Outlook}

Above we have tried to develop a microscopic picture of the early stages of quantum dot formation. At the fundament of our knowl- 
edge lies an understanding of the atomistic processes in epitaxial growth which are best explored through suitably conducted first-principles DFT calculations. In this contribution, we have attempted to demonstrate the application of the latter to the specific problems of surface atomic structure and adatom diffusivity in $\operatorname{InAs} / \mathrm{GaAs}(001)$ heteroepitaxy. From the typically used growth temperatures and the calculated energy barriers for diffusion, we conclude that the time and length scales involved in quantum dot formation in InAs heteroepitaxy on $\operatorname{GaAs}(001)$ span a few orders of magnitude. The importance of a wide range of length and time scales is a very general phenomenon in epitaxial growth. It is therefore clear that the theoretical description of this phenomenon requires more than a single theoretical tool. DFT calculations must be complemented by calculations on larger scales using elasticity theory or analytical interatomic potentials. Kinetic Monte Carlo simulations are able to integrate input form various sources, and enable us to bridge the gap between the atomic scale and experimentally relevant scales. The results from our first-principles calculations constitute important input to such kinetic Monte Carlo simulations. The feasibility of this method has already been demonstrated for homoepitaxy of GaAs [15, 46]. A typical problem where its usage is indispensable is the treatment of structural disorder in the wetting layer, observed in some samples. Future research will therefore be focused on kinetic Monte Carlo simulations of In diffusion on a disordered wetting layer and possible consequences for QD growth.

\section{Acknowledgments}

This work was supported by the Deutsche Forschungsgemeinschaft within the Sonderforschungsbereich 296. E. Penev would like to acknowledge fruitful discussions with F. Montalenti, O. Kirfel, I. Goldfarb, P. Machnikowski, and G. Goldoni during the NATO ARW on Quantum Dots: Fundamentals, Applications, Frontiers.

\section{References}

[1] D. Bimberg, M. Grundmann, and N. N. Ledentsov. Quantum dot heterostructures (Wiley, 1998).

[2] M. Kotrla, N. Papanicolaou, D. D. Vvedensky, and L. T. Wille, editors. Atomistic Aspects of Epitaxial Growth (Kluwer, Dordrecht, 2002).

[3] I. N. Stranski and L. Krastanov. Zur Theorie der orientierten Ausscheidung von Ionenkristallen aufeinander. Sitzungsber. Akad. Wiss. Wien, Math.-naturwiss. Kl. IIb 146, 797-810, 1938.

[4] M. Sugawara, editor. Self-assembled InGaAs/GaAs quantum dots (Academic, New York, 1999). 
[5] B. A. Joyce and D. D. Vvedensky. Mechanisms and anomalies in the formation of InAs-GaAs(001) quantum dot structures, in Atomistic Aspects of Epitaxial Growth, edited by M. Kotrla and N. Papanicolaou and D. Vvedensky and L. Wille (Kluwer,, Dordrecht, 2002), pp. 301-325.

[6] T. R. Ramachandran, R. Heitz, P. Chen, and A. Madhukar. Mass transfer in Stranski-Krastanov growth of InAs on GaAs. Applied Physics Letters 70: 640642, 1997.

[7] P. Ruggerone, C. Ratsch, and M. Scheffler. Density-functional theory of epitaxial growth of metals, in Growth and Properties of Ultrathin Epitaxial Layers, edited by D. A. King and D. P. Woodruff (Elsevier, Amsterdam, 1997) pp. 490-544.

[8] P. Kratzer, C. Morgan, and M. Scheffler. Density-functional theory studies on microscopic processes of GaAs growth. Prog. Surf. Sci. 59: 135-147, 1998.

[9] For a large number of examples the reader is referred to the following URL: http://www.fhi-berlin.mpg.de/th/paper.html.

[10] A. Kley. Theoretische Untersuchungen zur Adatomdiffusion auf niederindizierten Oberflächen von GaAs (Wissenschaft \& Technik Verlag, Berlin, 1997).

[11] C. G. Van de Walle and J. Neugebauer. First-principles surface phase diagram for hydrogen on GaN surfaces. Phys. Rev. Lett. 88: art. no. 066103, 2002.

[12] A. A. Stekolnikov, J. Furthmüller, and F. Bechstedt. Absolute surface energies of group-IV semiconductors: Dependence on orientation and reconstruction. Phys. Rev. B 65: art. no. 115318, 2002.

[13] K. Reuter and M. Scheffler. First-principles thermodynamics for oxidation catalysis: Surface phase diagrams and catalytically interesting regions. Phys. Rev. Lett. 90: art. no. 046103, 2003.

[14] F. Bechstedt. Principles of Surface Physics (Springer, Berlin, 2003).

[15] P. Kratzer, E. Penev, and M. Scheffler. Understanding the growth mechanisms of GaAs and InGaAs thin films by employing first-principles calculations. Appl. Surf. Sci. 216: 436-446, 2003.

[16] R. J. Needs, R. M. Martin, and O. H. Nielsen. Total-energy calculations of the structural properties of the group-V element arsenic. Phys. Rev. B 33: 37783784 (1986).

[17] M. Bockstedte, A. Kley, J. Neugebauer, and M. Scheffler. Density-functional theory calculations for poly-atomic systems: Electronic structure, static and elastic properties and ab initio molecular dynamics. Comp. Phys. Commun. 107: 187-222, 1997; URL: www.fhi-berlin.mpg.de/th/fhimd.

[18] K. Shiraishi. A new slab model approach for electronic-structure calculation of polar semiconductor surface. J. Phys. Soc. Jpn. 59: 3455-3458, 1996.

[19] V. P. LaBella, H. Yang, D. W. Bullock, P. M. Thibado, P. Kratzer, and M. Scheffler. Atomic structure of the $\operatorname{GaAs}(001)-(2 \times 4)$ surface resolved using scanning tunneling microscopy and first-principles theory. Phys. Rev. Lett. 83: 2989-2992, 1999.

[20] E. S. Penev. On the theory of surface diffusion in InAs/GaAs(001) heteroepitaxy (Technische Universität Berlin, 2002);

URL: www.fhi-berlin.mpg.de/th/publications/PhD-penev-2002 .pdf. 
[21] J. Tersoff, M. D. Johnson, and B. G. Orr. Adatom densities on GaAs: Evidence for a near-equilibrium growth. Phys. Rev. Lett. 78: 282-285, 1997.

[22] P. Kratzer, C. G. Morgan, and M. Scheffler. Model for nucleation in GaAs homoepitaxy derived from first principles. Phys. Rev. B 59: 15246-15252, 1999.

[23] C. T. Foxon and B. A. Joyce. Interaction kinetics of $\mathrm{As}_{2}$ and Ga on $\{100\}$ GaAs surface. Surf. Sci. 64: 292-304, 1977.

[24] M. Scheffler and P. Kratzer. Ab initio thermodynamics and statistical mechanics of diffusion, growth, and self-assembly of quantum dots, in Atomistic Aspects of Epitaxial Growth, edited by M. Kotrla and N. Papanicolaou and D. D. Vvedensky and L. T. Wille (Kluwer, Dordrecht, 2002) pp. 355-369.

[25] S.-H. Lee, W. Moritz, and M. Scheffler. GaAs(001) surface under conditions of low As pressure: Evidence for a novel surface geometry. Phys. Rev. Lett. 85, 3890-3893, 2000.

[26] C. Ratsch, W. Barvosa-Carter, F. Grosse, J. H. G. Owen, and J. J. Zinck. Surface reconstructions for $\operatorname{InAs}(001)$ studied with density-functional theory and STM. Phys. Rev. B 62: R7719-R7722, 2000.

[27] W. G. Schmidt, S. Mirbt, and F. Bechstedt. Surface phase diagram of $(2 \times 4)$ and $(4 \times 2)$ reconstructions of GaAs(001). Phys. Rev. B 62: 8087-8091, 2000 .

[28] W. G. Schmidt. III-V compound semiconductor (001) surfaces. Appl. Phys. A 75: 89-99, 2002.

[29] E. Penev, P. Kratzer, and M. Scheffler. (unpublished)

[30] C. Ratsch. Strain-induced change of surface reconstructions for InAs(001). Phys. Rev. B 63: art. no. 161306(R), 2001.

[31] A. Ohtake, J. Nakamura, S. Tsukamoto, N. Koguchi, and A. Natori. New structure model for the $\operatorname{GaAs}(001)-\mathrm{c}(4 \times 4)$ surface. Phys. Rev. Lett. 89: art. no. 206102, 2002.

[32] W. Haiss. Surface stress of clean and adsorbate-covered solids. Rep. Prog. Phys. 64: 591-648, 2001.

[33] D. I. Westwood, Z. Sobiesierski, E. Steimetz, T. Zettler, and W. Richter. On the development of $\mathrm{InAs}$ on $\mathrm{GaAs}(001)$ as measured by reflectance anisotropy spectroscopy: Continuous and islanded films. Appl. Surf. Sci. 123/124: 347351, 1998.

[34] T. Kita, O. Wada, T. Nakayama, and M. Murayama. Optical reflectance study of the wetting layer in (In, Ga)As self-assembled dot growth on GaAs(001). Phys. Rev. B 66: art. no. 195312, 2002.

[35] J. G. Belk, C. F. McConville, J. L. Sudijono, T. S. Jones, and B. A. Joyce. Surface alloying at InAs-GaAs interfaces grown on (001) surfaces by molecular beam epitaxy. Surf. Sci. 387: 213-226, 1997.

[36] Y. Garreau, K. Aïd, M. Sauvage-Simkin, R. Pinchaux, C. F. McConville, T. S. Jones, J. L. Sudijono, and E. S. Tok. Stoichiometry and discommensuration on $\operatorname{In}_{x} \mathrm{Ga}_{1-x} \mathrm{As} / \mathrm{GaAs}(001)$ reconstructed surfaces: A quantitative x-ray diffusescattering study. Phys. Rev. B 58: 16177-16185, 1998.

[37] A. G. de Oliveira, S. D. Parker, R. Droopad, and B. A. Joyce. A generalized model for the reconstruction of $\{001\}$ surfaces of III-V compound semiconductors based on a RHEED study of InSb(001). Surf. Sci. 227: 150-156, 1990. 
[38] M. Sauvage-Simkin, Y. Garreau, R. Pinchaux, M. B. Véron, J. P. Landesman, and J. Nagle. Commensurate and incommensurate phases at reconstructed (In, Ga)As(001) surfaces: X-ray diffraction evidence for a composition lock-in. Phys. Rev. Lett. 75: 3485-3488, 1995.

[39] J. G. Belk, J. L. Sudijono, D. M. Holmes, C. F. McConville, T. S. Jones, and B. A. Joyce. Spatial distribution of In during the initial stages of growth of InAs on GaAs(001)-c(4× 4). Surf. Sci. 365: 735-742, 1996.

[40] B. A. Joyce, D. D. Vvedensky, G. R. Bell, J. G. Belk, M. Itoh, and T. S. Jones. Nucleation and growth mechanism during MBE of III-V compounds. Mater. Sci. Eng. B 67: 7-16, 1999.

[41] D. J. Fisher. The Meyer-Neldel Rule. (Trans Tech Publications, Uetikon-Zürich, 2001).

[42] E. Penev, P. Kratzer, and M. Scheffler. Effect of strain on surface diffusion in semiconductor heteroepitaxy. Phys. Rev. B 64: art. no. 085401, 2001.

[43] H. T. Dobbs, D. D. Vvedensky, A. Zangwill, J. Johansson, N. Carlsson, W. Seifert. Mean-field theory of quantum dot formation. Phys. Rev. Lett. 79: 897-900, 1997.

[44] H. T. Dobbs, A. Zangwill, and D. D. Vvedensky. Nucleation and growth of coherent quantum dots: a mean field theory, in Surface Diffusion: Atomistic and Collective Processes, edited by M. Tringides (Plenum, New York, 1998) pp. 263-275.

[45] J. Tersoff and R. M. Tromp. Shape transition in growth of strained islands: Spontaneous formation of quantum wires. Phys. Rev. Lett. 70: 2782-2785, 1993.

[46] P. Kratzer, E. Penev, and M. Scheffler. First-principles studies of kinetics in epitaxial growth of III-V semiconductors. Appl. Phys. A 75: 79-88, 2002.

[47] A. Madhukar. A unified atomistic and kinetic framework for growth front morphology evolution and defect initiation in strained epitaxy. J. Cryst. Growth 163: 149-164, 1996.

[48] H. M. Koduvely and A. Zangwill. Epitaxial growth kinetics with interacting coherent islands. Phys. Rev. B 60: R2204-R2207, 1999. 